\title{
ESR MEASUREMENTS ON THE POLYMERIZATION OF LIQUID SELENIUM
}

\author{
D. C. KONINGSBERGER, J. H. M. C. VAN WOLPUT and P. C. U. RIETER \\ Department of Physics, University of Technology. \\ Eindhoven, The Netherlands
}

Received 23 November 1970

\begin{abstract}
In an earlier communication FSR measurements on de-oxygenized seletium were announced. The present paper zeports on further ESR measurements on liquid de-oxygenized selenium carried out in a temperature interval $240^{\circ} \mathrm{C}<T<420^{\circ} \mathrm{C}$. The $g$-value of the ESR signal is found to be $2.03 \pm 0.02$. The temperature dependence of the intensity and linewidth are interpreted as caused by a polymerization process. The heat of scission selenium-selenium bonds in chains was calculated to be $29 \pm 3 \mathrm{kcal} / \mathrm{mole}$. It seems that this is the first time, that ESR signals from pure Iiquid selenium have been reported.
\end{abstract}

\section{EXPERIMENTAL}

The ESR measurements were carried out using an E-15 Varian spectrometer with 2 Varian dual sample cavity, modified for performing measurements at high temperatures without loss in sensitivity. To obtain interpretable results at each temperature the signal of the empty cavity had to be subtracted from the selenium ESR recording.

Samples were made from selenium pellets, commercially obtained from Johnson and Matthey (catalogue nr JM 781, purity 1 in $10^{5}$ ). The pellets were evacuated at $10^{-5}$ torr for one hour at $20^{\circ} \mathrm{C}$, and were then heated in vacuum at a rate of $15^{\circ} \mathrm{C} / \mathrm{hr}$ until a temperature of $190^{\circ} \mathrm{C}$ was reached. The selenium was then outgassed and distilled at approximately $10^{-5}$ torr for 2 hours in the liquid state $\left(\approx 350^{\circ} \mathrm{C}\right)$. When this purification was finished the samples were sealed in quartz ESR sample tubes which had no detectable paramagnetic impurities.

\section{THEORY}

To describe the polymerization equilibrium the following reaction can be chosen ${ }^{*}$ :

$$
\begin{aligned}
& \mathrm{Se}_{8}^{\mathrm{R}} \rightleftharpoons \mathrm{Se}_{8}^{*} ; \quad K_{1}=\left[\mathrm{s}_{8}^{*}\right] /\left[\mathrm{Se}_{8}^{\mathrm{R}}\right], \\
& \operatorname{Se}_{(i+j)}^{*} \stackrel{\sim}{\sim} \mathrm{Se}_{i}^{*}+\mathrm{Se}_{j}^{*} ; \mathrm{K}_{2}=\left[\mathrm{s}_{i}^{*}\right]\left[\mathrm{s}_{j}^{*}\right] /\left[\mathrm{s}_{i+j}^{*}\right],(2)
\end{aligned}
$$

where

$$
\begin{aligned}
& \mathrm{Se}_{i}^{*}=\text { selenium diradical with } i \text { atoms; } \\
& \operatorname{Se}_{8}^{\mathbf{R}}=\text { selenium ring molecule with } 8 \text { atoms. }
\end{aligned}
$$

From the polymerization theory for sulphur developed by Gee [1] the following formula for the temperature dependence of the number of chainends (free radicals) can be derived:

$$
\ln \left(\frac{N_{S}}{2 M_{0} \varphi^{1 / 2}}\right)=-\frac{\Delta H_{2}}{2 R T}+\frac{\Delta S_{2}}{2 R},
$$

where $N_{\mathrm{S}}$ is the concentration of free radicals (kmole/kg), $\varphi$ the weight fraction polymer, $M_{0}$ the concentration of selenium atoms $(\mathrm{kmole} / \mathrm{kg})$, $\Delta H_{2}$ the heat of reaction (2) (heat of scission), and $\Delta S_{2}$ the entropy change of reaction (2).

\section{RESULTS}

As Wustrated by fig. 1 the linewidth $(\Delta H)$ found in selenium is very large. Because the spin concentration $\left(N_{\mathrm{S}}\right)$ is comparable to that in liquid sulphur, it is found that the signal amplitude $(A)$ is extremely small $\left[A=C N_{\mathrm{S}}(\Delta H)^{-2}\right]$. For this reason broad cavity signals must be carefully eliminated.

The average $g$-value is found to be $2.03 \pm$ 0.02 .

- $K_{2}$ is identical to $K_{5}$, used by Gee [II. 


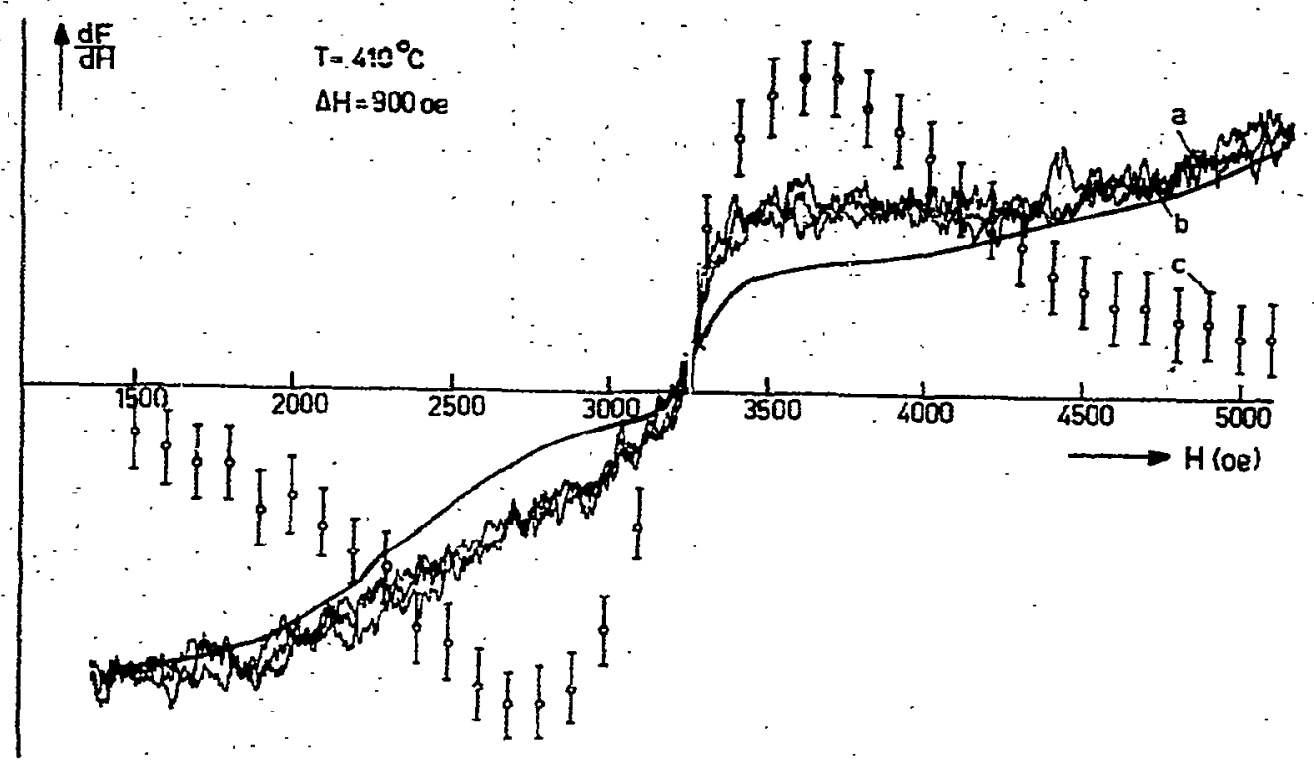

Fig. 1. a) ESR spectrum of liquid selenium superimposed on the cavity background signal. b) Cavity background signal; noise averaged by hand. c) The dotted points give the difierence ( $x$ ) between curve (a) and (b) (selenium EsR signat).
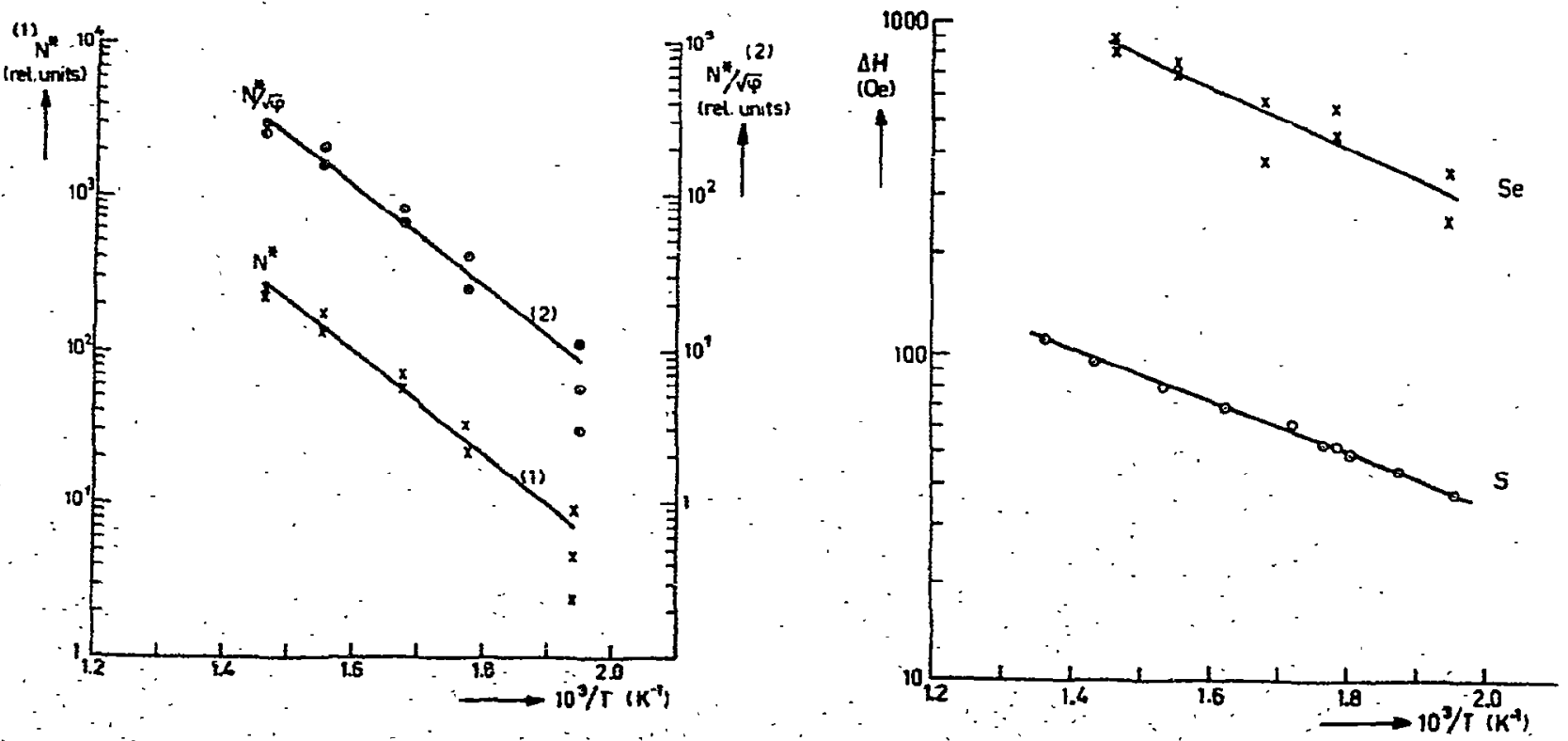

Fig. 2. Relative concentration of free radical $\mathrm{B}$ in liquid selenium. $N^{*}$ (curve 1) and $N^{*} / \varphi I / 2$ (curve 2 )

Fig. 3. Linewidth (AET) versus 1000/T of ESR spectra versus $1000 / T$. The straight line (eurve 2 ) was calcuof liquid selenium $(X)$ and aulphur $(O)$. The data for lated with the method of least squares. Curve 1 was sulphur were obtained from rêf. [4]. determined with help of curve $\dot{2}$. 
In fig. $2 N^{*}$ and $N^{*} / \varphi^{1 / 2}$ are given as a function $1000 / T$, where $N^{*}$ is the relative concentration of free radicals, which is proportional to $N_{\mathrm{S}} / 2 M_{0}$. Values for the weight fraction polymer $(\varphi)$ were obtained from data given by Briegleb [2]. From the slope of the straight line (2) in

fig. 2 we obtain a heat of scission $\Delta H_{2}=29 \pm 3$ $\mathrm{kcal} / \mathrm{mole}$.

In fig. 3 the linewidth of the ESR signals of liquid selenium and liquid sulphur are given as a function of 1000/T. Gardner and Fraenkel [3] proposed that the finite lifetime of the sulphur chainend spinstate is determined by the radical displacement reaction:

$$
\cdots s_{i}^{*}+\cdots s_{j}-s_{k} \cdots \Rightarrow \cdot s_{j}^{*}+\cdots s_{i}-s_{k} \cdots \cdot
$$

A similar mechanism is consistent with our ESR measurements on liquid selenium. In both cases the slope of the straight line gives the activation energy of the reaction involved. The activation energy is in both cases nearly the same. It may however be noted that the pre-exponential factor differs considerably. The evaluation of this infor mation concerning entropy change will be postponed until the number average chainlength has been determined by absolute spin calibration measurements.

\section{ACKNOWLEDGEMENTS}

The authors wish to acknowledge the stimulating discussions with Professor J. Cowen, Professor P.v.d. Leeden and Professor G. Schuit.

\section{REFERENCES}

[1] G.Gee. Trans. Faraday Soc. 48 (1952) 515.

[2] G. Briegleb, Z. Physik. Chem. Al4f (L929) 321.

[3] D. M.Gardiner and G. K. Fraenkel. J. Am. Chem. Soc. 78 (1956) 3279.

[4] D. C. Koningsberger and T. de Neef, Chem. Phys. Letters 4 (1970) 615. 